Article

\title{
Entre nature et culture : la nécessaire reconnaissance des territoires de très faible densité au Québec et au Canada
}

\author{
Martin Simard ${ }^{1}$ 凶 \\ ${ }^{1}$ Université du Québec à Chicoutimi, Département des sciences humaines et sociales, Canada
}

\begin{abstract}
Résumé. Le Québec, l'ensemble du Canada, voire l'Alaska ou la Russie, contiennent de vastes espaces nordiques inhabités. Cette affirmation, qui tombe sous le sens pour la majorité des Québécois et de leurs voisins anglophones, est pourtant lourde d'a priori discutables issus de la culture occidentale. En réalité, ces territoires sont soit occupés, parcourus ou nommés par les autochtones, depuis des siècles. Ces formes d'appropriation relativement légères, du moins en termes d'empreinte écologique, remettent en question le concept d'écoumène, notamment à cause de sa dimension binaire. Dans ce contexte, cette article vise à discuter des fondements du concept d'écoumène et, par ricochet, de nature sauvage (wilderness). Il apparaît évident que le terme écoumène est utilisé de manière réductrice et qu'il faille l'adapter pour décrire les divers modes possibles d'habiter le monde, notamment en milieu nordique. Au final, nous prônons la nécessaire reconnaissance des territoires de très faible densité, comme les espaces nordiques, afin d'entretenir des relations harmonieuses avec les autochtones.

Mots clés : Écoumène, densité, autochtones, Nord, Québec, Canada.
\end{abstract}

\section{Introduction}

Le Québec, l'ensemble du Canada, voire l'Alaska ou la Russie, contiennent d'immenses espaces nordiques inhabités. Cette affirmation, qui tombe sous le sens pour la majorité des Québécois et leurs voisins anglophones, est pourtant lourde d'a

CORRESPONDANCE :

\msimard@uqac.ca
HISTOIRE DE L'ARTICLE :

Reçu : 18 Septembre 2018

Accepté : 26 Septembre 2018

Disponible en ligne : 10 Octobre 2018 
priori discutables issus de la culture occidentale. En fait, ces territoires sont soit occupés, parcourus ou nommés par les autochtones, depuis des siècles (Auger, 2012). Ces formes d'appropriation relativement légères, du moins en termes d'empreinte écologique, remettent en question le concept d'écoumène, notamment à cause de sa dimension binaire. Au-delà de son caractère réducteur, l'expression latine associée de terra nullius, qui décrit les espaces hors-écoumène ou, par glissement politique et juridique, les espaces jugés sans-maîtres, a justifié l'asservissement et la dépossession totale ou partielle des populations autochtones en Amérique du Nord, et ailleurs dans le monde, par les puissances européennes et les nouveaux pays qu'elles ont engendrés (Paksy, 2016).

Malgré quelques adaptations illustrées par les expressions écoumènes discontinu et sporadique (De Koninck, 2009), la géographie humaine des espaces de faible densité ou de mobilité n'est pas correctement outillée par la nomenclature existante, et ce, en dépit de l'existence d'un courant humaniste et des nombreuses réflexions théoriques et épistémologiques sur les représentations spatiales et la territorialité (Lévy, 2008 ; Bédard, 2016 et 2017 ; Debarbieux, 2014). Il y a bien la notion de nordicité, conçue par le célèbre géographe québécois Louis-Edmond Hamelin (1975 et 2000), notion qui présente la particularité de se décliner par intensité, mais celle-ci ne prend acte des populations nordiques que de manière marginale, comme l'un des multiples éléments qui composent la réalité du Nord. En conséquence, elle ne constitue pas un substitut au terme écoumène, malgré ses vertus $^{2}$. Par ailleurs, avec l'anthropisation à large échelle de la planète et les changements climatiques, l'opposition entre l'écoumène, c'est-à-dire l'espace habité, et le milieu naturel, tend à disparaître car « il n'existe plus de zones échappant à toute influence humaine » (Baud et al., $2013: 310$ ).

Dans ce contexte, notre texte vise à discuter des fondements du concept d'écoumène et, par ricochet, de celui de nature sauvage, fréquemment appelé wilderness. Cette discussion nous aidera à préciser la portée de concepts géographiques de même qu'à mieux saisir l'originalité du peuplement des espaces nordiques et les malentendus ou paradoxes liés aux relations interculturelles. Il apparaît évident que le terme écoumène est utilisé de manière simpliste et qu'il faille l'adapter pour décrire les divers modes possibles d'habitation de la terre. Â l'image de la désuétude progressive des qualificatifs urbain et rural, et compte tenu également des problèmes récurrents de sa délimitation (Simard, 2012), le terme écoumène est peut-être en voie d'être relégué aux oubliettes. Outre son mépris intrinsèque des genres de vie autochtones, son inaptitude est encore accentuée par les

\footnotetext{
${ }^{2}$ La notion de nordicité mentale appelle à mieux comprendre, voire à adopter, les traditions et représentations des autochtones. Cependant, il ne remet pas à cause la définition ou la délimitation de l'écoumène québécois ou canadien.
} 
mouvements de main-d'œuvre vers les sites miniers isolés et les exploitations pétrolières off-shore. Cette mobilité, comme la très faible densité des territoires périphériques et divers autres facteurs, remet en cause la conception dominante de l'habiter (Berque, 2010). Et qu'en est-il des fameux parcs nationaux nord-américains ? Vus comme la quintessence de la nature sauvage préservée, ils sont, en réalité, passablement aménagés (routes d'accès, kiosques d'accueil ou d'hébergement, sentiers balisés, etc.), sans compter le fait qu'ils fassent souvent l'objet de manipulations fauniques ou floristiques et que l'on puisse trouver des propriétés privées enclavées à l'intérieur de ceux-ci (Moumaneix, 2012).

Notre réflexion s'appuiera sur une recension des écrits, des expériences découlant de visites de terrain antérieures en milieu nordique ainsi que de quelques données statistiques et supports cartographiques. Malheureusement, la revue des écrits peut difficilement être exhaustive considérant la masse de textes scientifiques et littéraires sur les thèmes apparentés de la nature, de l'environnement, des paysages, de l'écoumène, du territoire, etc. Néanmoins, le croisement de connaissance sur les expressions écoumène et nature sauvage pourrait apporter des réflexions originales sur la situation et le devenir des espaces nordiques. En ce qui concerne le cheminement des idées, nous tenterons d'abord de définir et de circonscrire les concepts d'écoumène et de nature sauvage. Par la suite, nous discuterons de la situation du Nord québécois et canadien, de son habitation caractéristique des espaces de très faible densité de même que des répercussions de son classement comme écoumène ou nature sauvage. Finalement, nous verrons que le mythe de la nature sauvage affecte également les territoires infra-écouménaux nord-américains, par le biais de l'étalement urbain, tout en contribuant à une certaine folklorisation des autochtones et des milieux nordiques, à travers le tourisme d'aventure.

\section{1. Écoumène : considérations étymologique et épistémologique}

Le concept d'écoumène est très ancien, remontant aux travaux fondateurs d'Hérodote, le père de l'histoire et de la géographie (Claval, 2011). Aux yeux de plusieurs, il s'agit même de l'objet central de la géographie humaine, voire de la géographie dans son ensemble. Cette conception de l'écoumène comme thème prépondérant de la discipline s'est maintenue, de Strabon à Sorre en passant par Vidal de La Blache, évoluant d'une vision ethnocentrique à une approche plus « cosmopolite » (Tessier, 2004). Après une mise de côté tributaire de l'essor de la géographie quantitative, qualifiée dans les années 1960 et 1970 de « nouvelle géographie ", le terme a retrouvé une place de choix dans la discipline, notamment grâce aux travaux d'Augustin Berque (1990, 1996, 2000, 2010 et 2011). Toutefois, l'écoumène prend dès lors un sens renouvelé qui s'inscrit dans l'approche phénoménologique et la géographie humaniste. Nous y reviendrons plus loin. 
Tirant donc son origine de la Grèce antique, le terme écoumène peut également s'écrire œkoumène ou œcoumène. De manière pragmatique, ce concept réfère essentiellement à l'espace humanisé ou habité, la " maison » de l'homme au sens large (Baud et al., 2013 : 166). Ce territoire transformé par l'humain à divers dégrés, qui transgresse l'opposition urbain-rural, était nécessairement appelé à prendre de l'expansion avec le temps et la croissance démographique. Par moment, on parle plutôt d'espace habitable plutôt qu'habité mais la distinction devient ténue dans un monde de 7,5 milliards d'individus (Brunet et al., 2009). Quoi qu'il en soit, pour les esprits cartésiens, l'écoumène est difficile à délimiter à toutes les époques : doit-on y intégrer seulement les villes et les villages ? Si l'on tient compte des zones adjacentes aux noyaux de peuplement, faut-il se restreindre aux espaces agricoles, inclure les réseaux de circulation ou prendre acte des territoires dotés d'administrations locales ou d'un morcellement foncier permettant la propriété privée du sol ? Et que penser des espaces lacustres ou maritimes fortement fréquentés, des sites religieux, militaires, industriels ou touristiques isolés, voire des stations scientifiques ou météorologiques en zones de climat extrême?

À l'origine, le concept d'écoumène exclut d'emblée tout territoire aux populations non sédentarisées, laissant de côté un large pan de l'humanité. Les « barbares " extérieurs au monde hellénistique, et éventuellement romain, ne méritaient guère plus de considération avec leurs établissements humains dispersés et de petite taille ou leur genre de vie de nomades ${ }^{3}$ (Pollini, 2012). En dépit de la disparition presque complète des peuples nomades au XXIe siècle, il y a encore de grands espaces terrestres peu ou pas habités, des no man's land, soit les hautes montagnes de l'Himalaya, des Alpes et des Andes, la forêt amazonienne, les déserts du Sahara ou de Gobi, l'Antarctique ou le Groenland, pour n'en nommer que quelques-uns (Hertzog et al., 2011). Pourtant, mis à part la situation particulière de l'Antarctique, ces différents territoires sont occupés d'une certaine manière, ceux-ci ayant été nommés, fréquentés plus ou moins régulièrement, subtilement altérés, dans plusieurs cas (Mann, 2007). Surtout, ces milieux sont porteurs de sens pour les populations avoisinantes ou de passage, aussi peu nombreuses soient-elles.

Il apparaît progressivement évident à la majorité des observateurs de la « scène géographique » que les rapports à la nature et aux territoires, de même que leur catégorisation, relève de la culture. Au même titre que le concept de nature, l'écoumène serait une réalité construite « défini comme le rapport social au monde biophysique », pour paraphraser Jacques Lévy (2008: 39). On peut aller jusqu'à dire que la nature n'existait pas avant qu'on invente le terme en Occident, soit avant que l'humain ne se perçoive comme étant hors ou à côté de la nature et qu'il façonne des

\footnotetext{
3 Malgré l'ethnocentrisme du concept, Hérodote aurait fait preuve d'un certain universalisme en s'intéressant aux peuples des marges de l'écoumène.
} 
milieux anthropiques de plus en plus étendus et artificiels (Evernden, 1992). Depuis peu, moult travaux s'intéressent à la cartographie autochtone dans l'optique de redonner vie aux représentations anciennes ou actuelles des premières nations et de localiser celles-ci (Glon, 2012). Ces représentations sont fortement signifiantes, du moins pour les autochtones, car elles résultent en grande partie d'une vision holistique où la nature et l'humain sont inséparables.

Les recherches des dernières décennies sur l'écoumène vont dans le même sens à travers les réflexions d'Augustin Berque (1990, 1996, 2000, 2010 et 2011). Ce dernier affirme ceci : " l'écoumène peut se définir comme l'ensemble des milieux humains, chacun de ceux-ci étant la relation d'un groupe humain à son environnement » (Berque, 2012 : 237). L'écoumène se construit à travers le processus de "médiance », plus précisément par l'intermédiaire du mécanisme de « trajection » (1990 et 2000), mécanisme que l'on peut résumer simplement comme étant le passage d'un objet du monde objectif ou monde subjectif. C'est donc pratiquement la totalité de la planète qui est concernée car le critère adopté par Berque semble être l'existence d'une relation avec l'humain, c'est-à-dire, au-delà de son occupation ou de son exploitation comme ressource, que le territoire soit signifiant pour un groupe d'humains en ayant fait l'expérience. Appliquée au Nord québécois ou canadien, cette définition à saveur sociopsychologique tient davantage compte de la situation d'un peuplement superficiel sur de larges étendues, étendues pourtant empreintes de fortes charges symboliques, c'est-à-dire caractérisées par des points forts, des réseaux, des lieux aux significations et aux temporalités variées (Desbiens, 2015).

\section{Délimiter l'écoumène québécois et canadien}

Les enjeux de définition et de délimitation de l'écoumène sont toujours présents aujourd'hui lorsque vient le temps de cartographier celui-ci sur un territoire étatique quelconque. À titre d'exemple, l'organisme Statistique Canada a préféré éviter le sujet en classant d'abord bêtement tous les espaces non urbains d'un pays de 10000 $000 \mathrm{~km} 2$ dans la catégorie rurale, même les grandes aires de forêt boréale, de taïga et de toundra, ainsi que les îles inhospitalières de l'Arctique canadien (Statistique Canada, 2018). Ce rural, qui constitue plus de $90 \%$ de la superficie terrestre nationale, amalgame les territoires agricoles relativement restreints et les grands espaces peuplés de façon archipélagique, lesquels espaces sont traditionnellement exclus de l'écoumène. En effet, pour le voyageur, l'absence prolongée de terres agricoles est souvent synonyme d'une sortie d'écoumène, les paysages n'étant plus façonnés par l'humain de manière marquante. Rappelons ici que la densité de la région administrative du Nord-du-Québec est de 0,1 h. / $\mathrm{km} 2$ et de 0,03 h. / km2 dans la moitié nord de ce territoire que l'on nomme le Nunavik (ISQ, 2011). La moyenne 
québécoise est actuellement de 6,5 h. / $\mathrm{km} 2$ et celle de l'ensemble du Canada est de 3,9 h. / km2.

Avec les années, Statistique Canada a développé le concept plus fin de zones d'influence métropolitaine (ZIM). Ainsi, les unités territoriales standardisées (des " divisions de recensement » correspondant généralement aux frontières des municipalités locales) sont classées en fonction du niveau d'intégration de la population active à l'économie des villes de plus de 10000 habitants étant situées à proximité (voir la figure 1). Selon notre interprétation, l'étendue hors écoumène serait de la sorte équivalente aux plages de «ZIM nulles ", ce qui correspond grossièrement à $50 \%$ des terres sous juridiction canadienne. Si l'on ajoute la catégorie des « ZIM faibles » à la délimitation des milieux externes à l'écoumène, on gagne encore $10 \%$ à $15 \%$ du territoire. Des pays comme le Canada apparaissent alors comme largement inhabités et l'état particulier des régions nordiques demeure gommé par ces traitements statistiques et cartographiques conçus par et pour le monde urbain et industriel.

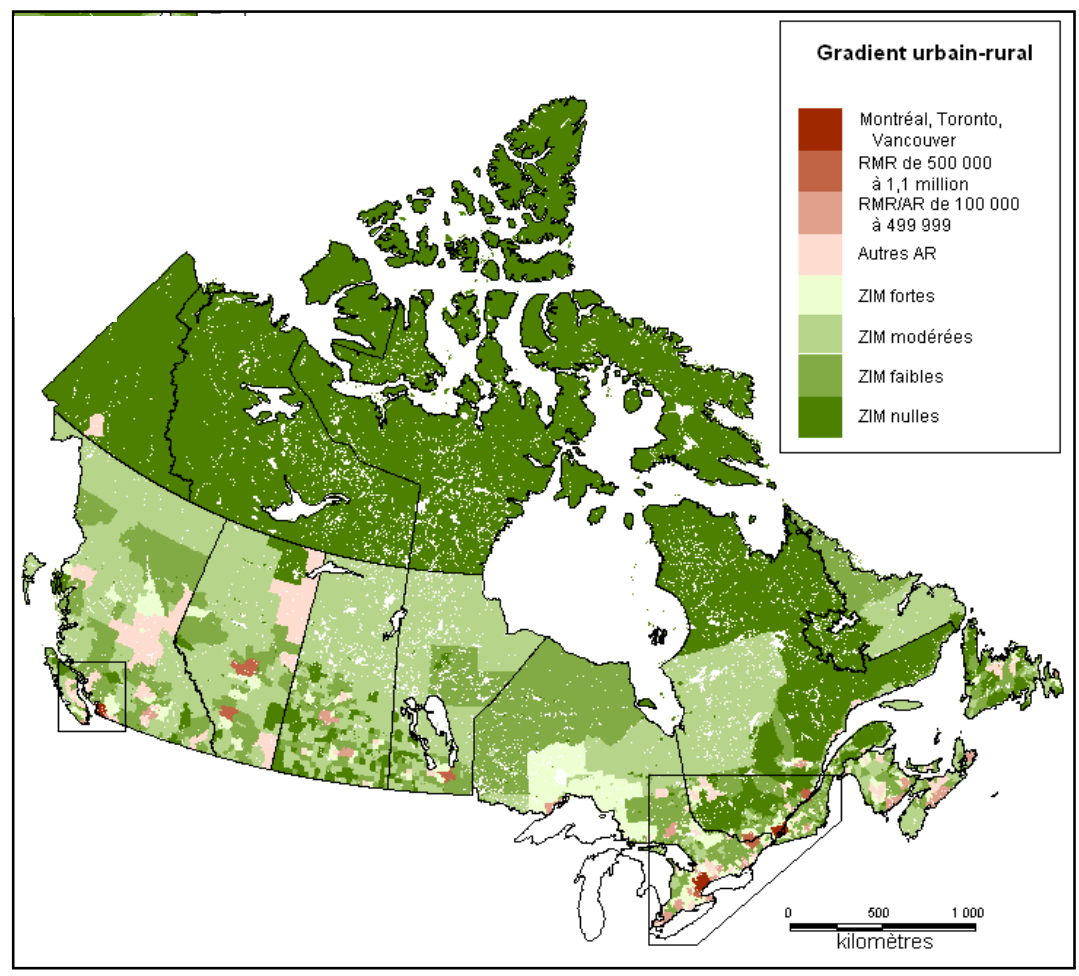

Figure 1 : Les espaces urbains et les zones d'influence métropolitaine au Canada

Certains spécialistes ont cherché à proposer un classement plus systématique de l'occupation du territoire au Canada. En effet, le géographe Rodolphe de Koninck dans son manuel Le monde à la carte (2009) a esquissé une classification de l'écoumène 
canadien. S'inspirant des travaux d'Hamelin, De Koninck catégorise l'espace national en fonction de la densité approximative des différentes régions, densités généralement tributaires de la marche du peuplement euro-canadien (voir les figures 2 et 3). Il regroupe dans l'écoumène les régions métropolitaines, les espaces agricoles, les réseaux de transport et les villes et villages périphériques de taille «significative». Bien qu'étant quelque peu bancale, cette proposition a le mérite d'être claire et pédagogique. Du plus, on y reconnaît les particularités des terres de très faible densité en proposant des concepts originaux (écoumène discontinu, écoumène sporadique et écoumène hydrographique). Au final, le milieu non écouménal se limite alors aux môles répulsifs continentaux ou insulaires, soit approximativement $20 \%$ à $25 \%$ du territoire canadien.

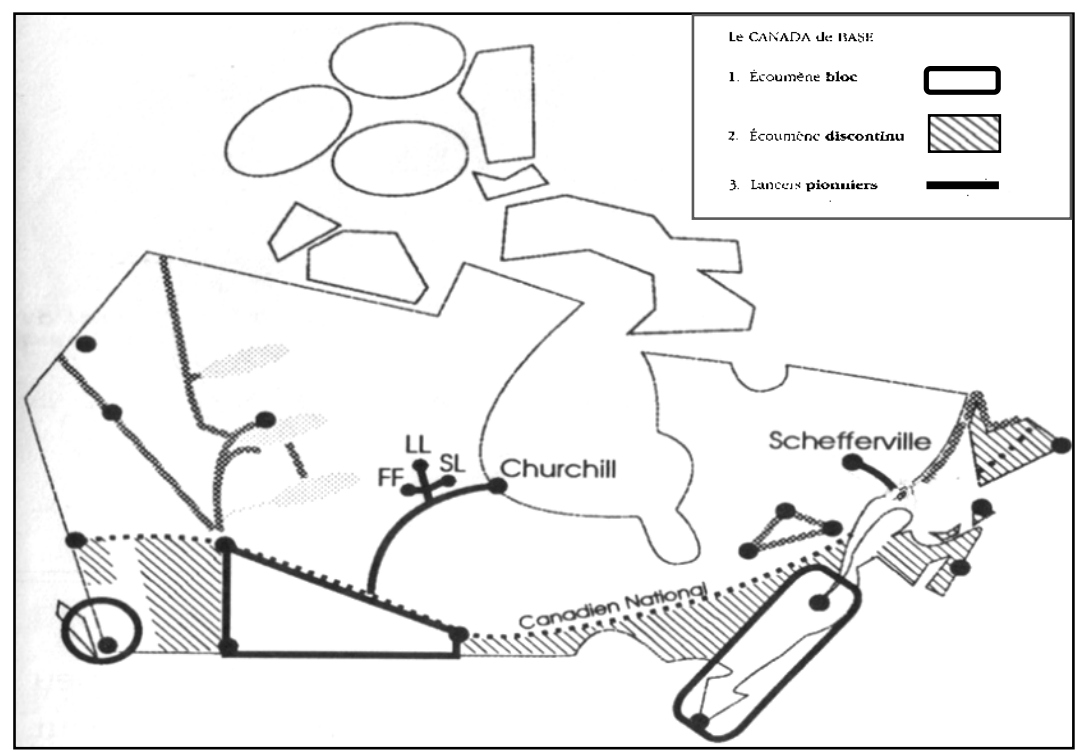

Figure 2 : Le Canada de base (source : De Koninck, 2009)

Pour sa part, la géographe Caroline Desbiens défend une conception de l'écoumène qui rende encore davantage justice aux espaces nordiques, du moins ceux intégrés aux pratiques et à l'univers culturel des autochtones du Québec et du Canada. Celle-ci énonce le principe suivant : " afin d'intégrer pleinement les territorialités autochtones, la notion d'écoumène doit être élargie jusqu'à englober l'héritage du nomadisme »(2012 : 645). Desbiens ne fait pas de proposition cartographique. Toutefois, elle souligne que la construction des installations hydroélectriques découlant du projet de la Baie James a ennoyé ou remodelé profondément de multiples territoires occupés ou utilisés fréquemment ou sporadiquement par les autochtones de la nation Crie. Cela s'est fait sans même que les gestionnaires et la population du Québec méridional ne soient conscients de 
l'espace vécu et des représentations spatiales des premières nations (voir la figure 4). Un autre exemple des rapports aux territoires des autochtones est celui des séjours des Innus du Québec dans la région septentrionale du Mushuau-nipi (Hamelin, 1973 ; Pollock, 2008 ; Schneider, 2013), voyage symbolique au cœur de la contrée sans arbres des caribous qui s'apparente au pèlerinage à La Mecque pour les musulmans, dans une certaine mesure.

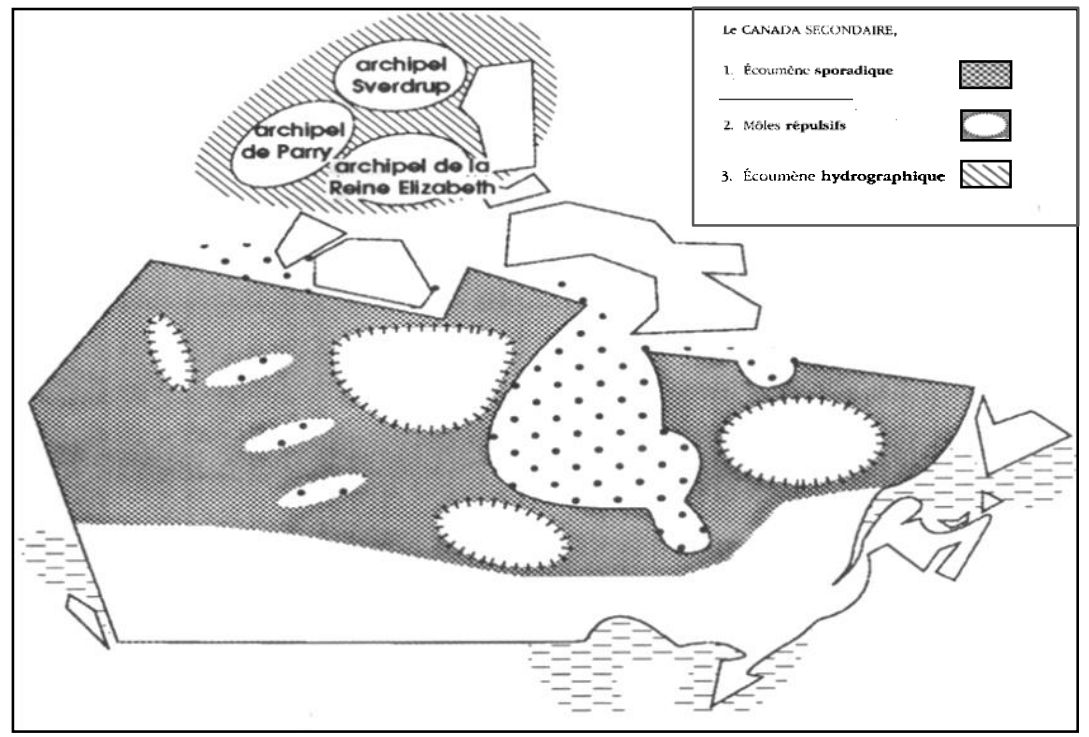

Figure 3 : Le Canada secondaire (source : De Koninck, 2009)

Cet " écoumène mental ou perceptuel » autochtone est encore assez peu connu ${ }^{4}$. Comme toute territorialité humaine, il serait le résultat d'un processus socioculturel qui ferait aussi ressortir une dialectique entre le territoire qui est mien et celui de l'autre ou des autres (Dorais, 2008), malgré l'existence probables de zones neutres, de lieux de rencontre et de formes d'appropriation partagée. En effet, les nations ou groupes autochtones avaient, et ont toujours dans plusieurs cas, des territoires de référence avec des frontières ou des aires de transition (voir la figure 5), même si ces territoires traditionnels n'ont généralement pas de statut légal particulier et qu'ils n'apparaissent que très rarement sur les cartes (Germain, 2012). Qui plus est, au sein même des différentes nations autochtones, les clans ou les familles ont souvent eus, ou ont toujours, des territoires exclusifs pour la chasse ou la trappe (Lacasse, 1996). Comme le présageait déjà Hamelin en 1975, cette territorialité se rapproche progressivement de celle des "Blancs ", ce qui se reflète dans les revendications territoriales des premières nations et des Inuit.

\footnotetext{
${ }^{4}$ L'Atlas des peuples autochtones du Canada (2018), édité par la Société géographique royale du Canada, apporte des témoignages enrichissants sur ce thème.
} 


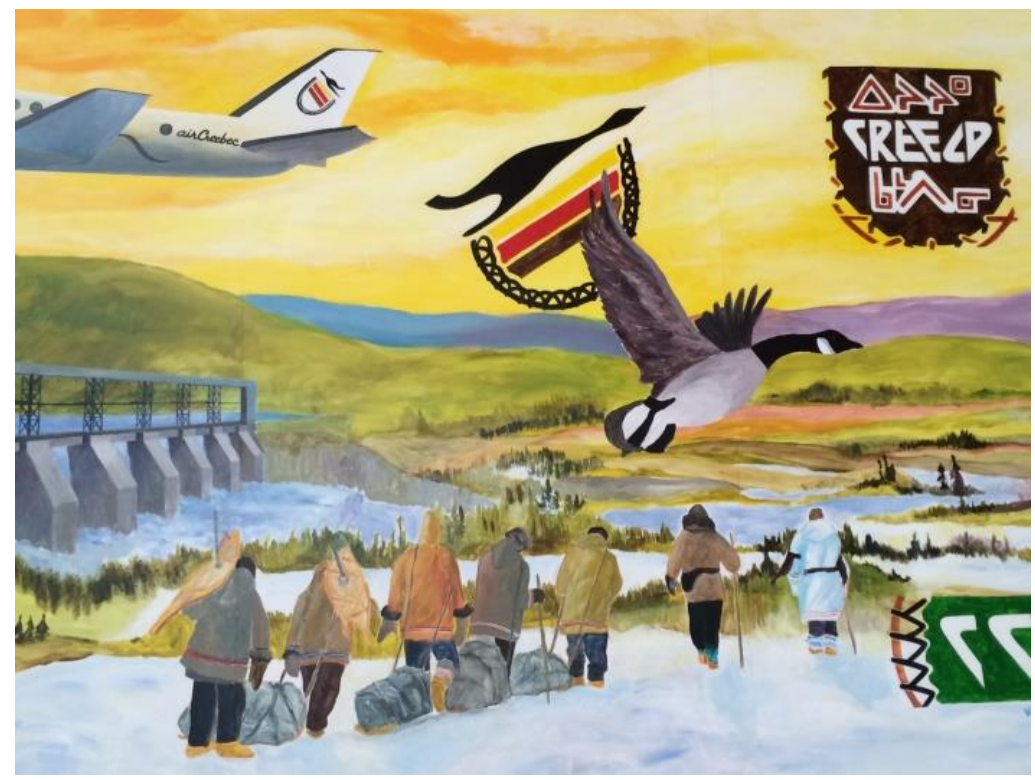

Figure 4 : Les autochtones et le projet de la Baie James (source : musée d’Oujé-Bougoumou)

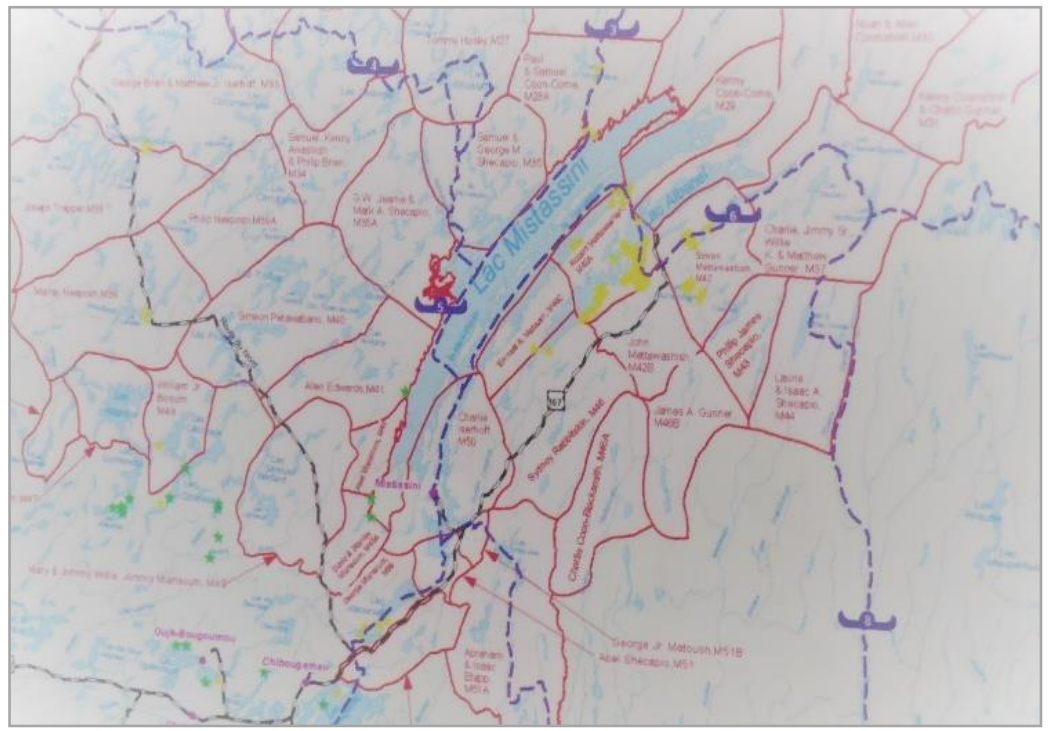

Figure 5 : Les aires de trappage autour du Lac Mistassini au Québec (photo. M. Simard) ${ }^{5}$

${ }^{5}$ L'attribution des aires de trappage dans la région de l'Eeyou Istchee-Jamésie a été parrainée par la Société de développement de la Baie James et Hydro-Québec. Cela ne contredit pas la tradition préexistante de répartition des aires de trappe ou de chasse chez les autochtones. 
En parallèle, l'évolution des représentations du Nord au sein de la population non-autochtone et la reconnaissance progressive de son écoumènabilité favorise une harmonisation des relations interculturelles. La prise en compte des avis et des intérêts des autochtones est plus forte que par le passé dans le développement du Nord québécois. Traités comme de vulgaires squatteurs lors de la planification des projets de la Baie James (Sioui, 2018), les autochtones ont exprimé leur présence sur la scène médiatique, ce qui a mené à la signature de la convention de la Baie James et $\mathrm{du}$ Nord québécois. Ils sont maintenant consultés et interpellés comme partie prenante par les politiciens et les industriels œuvrant dans le Nord, même si quelques nations semblent favorisées au détriment d'autres (Farget et al., 2014). Par exemple, les compagnies minières négocient régulièrement des ententes sur les retombées et les avantages (ERA) des projets miniers avec les communautés autochtones, processus qui n'est pas parfait mais qui peut devenir un outil intéressant lorsque bien utilisé (Knotsch et Fullum-Lavery, 2010). En dépit des vicissitudes du Plan Nord du gouvernement du Québec (Rivard et Desbiens, 2011), les autochtones apparaissent comme des acteurs incontournables, ne serait-ce que par leur potentiel de nuisance ou de blocage face aux projets industriels localisés dans le voisinage de leurs communautés. Les règles et instances administratives issues de la convention (1975), l'entente dite « de la paix des braves » (2002) et la récente création du gouvernement régional de l'Eeyou-Istchee-Baie-James (2014) sont des preuves tangibles que le Nord du Québec est de moins en moins envisagé comme une Terra Nullius.

\section{3. Écoumène, nature sauvage, milieux nordiques et autochtones}

L'expression nature sauvage, qualifiée de wilderness en contexte anglo-saxon, s'avère utile à aborder dans ce texte dans la mesure où elle s'appuie également sur une opposition homme-nature, et la spatialisation de cette dichotomie, de même qu'elle détermine l'usage réservé à la nature et la position des peuples autochtones au sein des sociétés occidentales. D'abord perçu comme l'opposé de l'écoumène, soit l'érème ou l'espace qui n'est pas habité ou qui est en-dehors de la maison de l'homme, la nature sauvage constitue une construction sociale à consonance géographique, au même titre que l'écoumène. Selon Joliet et Jacobs, « le wilderness désigne une relation particulière à la nature, caractérisée par des représentations et des pratiques paysagères qui lui sont propres. Il incarne un fond mythique dont la puissance évocatrice émane des grands espaces vierges de l'Amérique du Nord au XIXe siècle » (2009 : 27). Certains attribuent une origine étatsunienne à ce mythe et dénoncent son caractère impérialiste (Bourgeois, 2016), d'autres mettent en exergue sa participation à la consolidation de l'identité canadienne, notamment par le biais de l'érection de parcs nationaux (Craig-Dupont, 2012). 
Le Québec ne serait pas trop différent sur ce plan, si ce n'est l'émergence d'un mythe du Nord salvateur de la nation canadienne-française, de la fin du XIXe siècle jusqu'à la révolution tranquille (Morissonneau, 1978 ; Roche-Eley, 1979). Celui-ci aurait favorisé la colonisation agricole de régions du pré-Nord ou du Moyen-Nord québécois, des Laurentides à l'Abitibi, terres d'avenir pour les francophones à l'étroit dans la plaine du Saint-Laurent (Morissonneau, 1978). Les travaux de la Baie James seraient le prolongement non agricole de cet élan de mythification du Nord (Desbiens, 2015). Le Plan Nord (2011 et 2015) s'inspire en partie de cette vision. Toutefois, mondialisation oblige, il n'est plus question de faire migrer la population vers le Nord mais d'y expédier des travailleurs de passage selon le système de navettage aérien ou fly-in / fly-out. Le Nord est maintenant vu comme peuplé et la question autochtone est une préoccupation constante pour les gouvernements qui sont sensibles aux sorties médiatiques ou aux manifestations sur le terrain.

Quelle que soit son origine et l'aliénation culturelle qui résulterait de son emprunt, le concept de nature sauvage ou de wilderness percole largement les Amériques pour irriguer les pays occidentaux, voire l'ensemble des sociétés en cours de mondialisation. Posthumus (2012) étudie les nuances de ce transfert dans l'imaginaire environnemental français. Tant en Europe qu'en Amérique du Nord, la nature sauvage, autrefois milieu hostile réservé à quelques explorateurs intrépides, devient un espace de loisir pour un public de plus en plus large, le lieu d'expériences perçues comme puissantes et authentiques où l'individu cherche à trouver sa nature véritable et à dépasser ses limites. La culture populaire propage cette image du wilderness dans les films ou les livres à succès (Brereton, 2005). La conquête du milieu naturel n'est plus seulement une affaire de société, une responsabilité d'État ou l'apanage d'entreprises privées extractives ou énergétiques, il s'agit d'un projet individuel, d'une quête existentielle qui ne peut interpeller que le sujet engagé sur cette voie, mais qui découle de l'imaginaire collectif.

Cette glorification de la nature sauvage entraîne les interrogations suivantes : la maison de l'homme serait-elle en si mauvais état que l'on doit la quitter pour vivre pleinement et retrouver une forme d'authenticité ? Au contraire, serait-elle trop confortable pour une bonne partie de la population, dans nombre de pays ? Dans tous les cas, si d'aucns réclament une littérature qui soit moins anthropocentrique et permette un recentrement, la culture populaire semble faire fi de ce désir de changement (Blanc et al., 2008). La nature perçue comme sauvage stimule toujours l'imaginaire nord-américain et occidental comme endroit où la vie se révèle comme plus intense et, pour ainsi dire, plus vraie... que nature. Cependant, ce ne serait pas que le wilderness qui serait la cible de l'imaginaire collectif, le mythe pastoral intervient également dans l'aménagement interne de l'écoumène par le biais du modèle de la banlieue jardin et de l'habitat pavillonnaire, éléments fondamentaux du rêve américain, rêve qui est aussi largement canadien et québécois (Côté, 2015). Les 
grands parcs et jardins urbains ont aussi été créés pour que le citadin se ressource et retrouve un état de bien-être, dès l'époque de la ville industrielle (Mercier et Béthemont, 1998). On leur accorde ainsi de nombreuses vertus sanitaires ou morales toujours d'actualité avec le verdissement accentué propre à l'urbanisme durable.

Incidemment, l'attrait pour la nature sauvage peut être considéré comme un atout pour le Nord et ses paysages peu transformés. Le tourisme d'aventure peut ainsi être un instrument de développement territorial durable qui implique également une forme de reconnaissance culturelle pour les communautés autochtones, malgré ses aléas (Iankova, 2005). Dans cette optique, le gouvernement provincial, de concert avec l'Administration régionale Kativik, a récemment implanté de nouveaux parcs nationaux au Nord de la péninsule du Québec-Labrador, notamment ceux de Pingaluit ou de Tursujuq (Desroches et Freedman, 2012). Ces équipements sont administrés en partenariat avec les Inuit. Les visiteurs, relativement rares pour le moment, y vivent, sans doute, une expérience personnalisée qui relève de la rencontre purificatrice avec la nature sauvage.

Ces réflexions soulèvent une question centrale sur le statut de l'autochtone dans l'imaginaire des populations allochtones du Québec et du Canada : l'indien ou l'inuk est-il un élément de la nature sauvage ou, à l'inverse, est-il un signe de son écouménalité naissante ou assumée ? N'oublions pas que la loi canadienne sur les indiens traite ceux-ci comme des enfants (de la nature ?) sous la tutelle du gouvernement fédéral (Girard et Brisson, 2018). De plus, lors d'enquêtes récentes auprès de travailleurs blancs œuvrant périodiquement dans le Nord du Québec, la présence autochtone a paru indissociable de la perception et la représentation de l'environnement nordique (Simard et al., 2018). S'agit-il d'une représentation aux racines profondes ou, tout bonnement, un reflet de la concentration ou du poids démographique des autochtones dans les régions nordiques, contrairement aux régions d'origine de la majorité des travailleurs?

\section{Conclusion}

Influencé par le mythe américain de la nature sauvage, lequel prend quelques couleurs particulières en contextes canadien et québécois, le découpage de l'écoumène du Canada a longtemps ignoré les territoires autochtones en milieu nordique. Ce constat plutôt technique a eu des conséquences significatives sur les situations politique, juridique et socioéconomique des autochtones et la qualité des relations interculturelles. Des espaces considérés comme n'étant pas véritablement habités peuvent être accaparés, aménagés sans consentement, voire spoliés, par la majorité dominante dans des pays fondés sur un héritage colonial, comme le Canada. Ainsi, il apparaît nécessaire de reconnaître comme écouménal des territoires de très faible densité, tels les espaces nordiques, afin d'affiner les outils conceptuels des 
géographes et, surtout, d'entretenir des relations plus harmonieuses entre les blancs et les autochtones. Un développement territorial durable et culturellement adapté aux milieux nordiques est aussi conditionné par un changement de mentalité chez les allochtones, virage qui semble amorcé, du moins chez les intellectuels travaillant dans le champ des études nordiques ou de la nordicité.

Paradoxalement, les autochtones doivent également intérioriser complètement une image décolonisée d'eux-mêmes et nourrir la vision plus fine du territoire vécue par leurs ancêtres. Ce parcours est exigeant, tiraillés comme ils le sont généralement entre la survie de leurs traditions et l'insertion dans la modernité. À titre d'exemple, le tourisme est une activité économique qui soulève l'adhésion chez les habitants du Nord, étant donné qu'il exige des aménagements moins lourds que l'industrie et qu'il valorise le territoire et la culture autochtones, d'une certaine manière. Néanmoins, l'avènement du tourisme de croisières et des parcs nationaux pourrait participer à l'instrumentalisation des mythes de la nature sauvage et du bon sauvage. En particulier, les croisières dans l'Arctique, qui attirent des touristes en grand nombre dans les villages côtiers, sont fréquemment perçues comme intrusives. Vendre les grands espaces et encourager la folklorisation de la culture ? Serait-ce un moindre mal ? En parallèle, les emplois dans les mines et les installations hydroélectrique offrent un environnement de travail présentant plusieurs difficultés. Néanmoins, ces emplois ont le mérite de permettre des horaires de travail intermittents qui peuvent s'ajuster avec les pratiques traditionnelles de chasse et de pêche. Décidément, il n'y a pas de solution facile face à l'enjeu du développement social et économique des communautés autochtones. Il n'en demeure pas moins que reconnaître sans ambages les territoires de faible densité comme faisant partie intégrante de l'écoumène et les populations autochtones comme étant des composantes à part entière de la société globale, seraient assurément des pas en avant.

Outre les défis d'intégration au marché de l'emploi des autochtones, enjeu important car les communautés autochtones en milieux nordique ou plus méridionaux vivent des problèmes socioéconomiques majeurs, la question de l'égalité de leur traitement par les gouvernements supérieurs, notamment celui du Québec, est de mise. En effet, les nations crie, inuite et nascapie se sont vues attribuer un statut plus habiletant que les autres, notamment les Innus et les Anishnabés qui cotoïent les trois premières groupes, dans plusieurs cas à l'intérieur d'espaces nordiques de très faible densité. Pourquoi ces nations n'ont-elles pas de traités équivalents ou semblables à celui de la Convention de la Baie James et du Nord québécois ? Nommément, les négociations multipartites avec les Innus s'éternisent en vue de la création d'un éventuel Nitassinan. Est-ce le résultat d'un manque d'organisation ou de cohésion des différentes communautés appartenant à ces 
nations ou est-ce parce que celles-ci se rapprochent de l'écoumène traditionnel ou se retrouvent au cœur de celui-ci ? Y a-t-il un salut pour les collectivités autochtones québécoises et canadiennes localisées en-dehors de la nature perçue comme sauvage, c'est-à-dire étant situées dans un univers européanisé au sein duquel le sol est privatisé, les structures administratives locales et régionales bien établies et où les populations blanches majoritaires montrent peu d'ouverture à changer le statu quo ?

\section{Références}

Auger, R., 2012, "La présence ancestrale des paléoesquimaux au Nunavik », dans Girard, R., dir., Histoire du Nord-du-Québec, Québec, Presses de l'Université Laval, p. 39-68.

BAud, P., Bourgeat, S. et BrAS, C., 2013, Dictionnaire de la géographie, Paris, Hatier, pages, 607 pages.

BEDARD, M., 2017, « Les vertus identitaire, relationnelle et heuristique de la territorialité - D’une conception culturelle à une conceptualisation tripartite », Cybergeo : revue européenne de géographie, document 838 [En ligne] URL http://journals.openedition.org/cybergeo/28853, consulté le 07 septembre 2018.

BEDARD, M., 2016, « Réflexion sur les perceptions, conceptions, représentations et affections, ou la quadrature des approches qualitatives en géographie », Cahiers de géographie du Québec, vol. $60, \mathrm{n}^{\circ} 171$, p. 531-549.

Bourgeois, M.-J., 2016, Pour une sociologie du concept de wilderness : étude de la globalisation d'une représentation étasunienne de la nature, Mémoire de maîtrise en sociologie, Université du Québec à Montréal, 122 pages.

Berque, A., 2012, "Écoumène », dans Ghorra-Gobin, C., dir., Dictionnaire critique de la mondialisation, Paris, Armand Colin, p. 237-241.

BERQUE, A., 2011, « Le rural, le sauvage et l'urbain », Études rurales, n 187 [En ligne] URL : http://journals.openedition.org/etudesrurales/9367, consulté le 3 septembre 2018.

Berque, A., 2010, Histoire de l'habitat idéal, de l'Orient vers l'Occident, Paris, éditions du félin, 400 pages.

BERQUe, A., 2000, Écoumène. Introduction à l'étude des milieux humains, Paris, Belin, 271 pages.

BERQue, A., 1996, Être humains sur la terre, Paris, Gallimard, 212 pages.

BERQue, A., 1990, Médiance, de milieux en paysages, Montpellier, Reclus, 163 pages.

Blanc, N., Pughe, T. et Chartier, D., 2008, "Littérature et écologie : vers une écopoétique », Écologie et politique, vol. 36, $\mathrm{n}^{\circ} 2$, p. 15-28.

Brunet, R., Ferras, R. et Thery, H., 2009, Les mots de la géographie (3e éd., Paris, Reclus, 518 pages.

Brereton, P., 2004, Hollywood Utopia: Ecology in Contemporary American Cinema, Bristol - UK, Intellect Books, 160 pages.

Claval, P., 2011, Histoire de la géographie (3e éd., Paris, PUF, 128 pages.

COTE, M., 2015 La garnison et le jardin. De la lutte à l'étalement urbain au projet de rurbanisation : analyse du régime aménagiste québécois, thèse de doctorat, Département de géographie, Université Laval, 326 pages.

CRAIG-DuPONT, O., 2012, "Science gouvernementale et nation building : Parcs Canada et la réinterprétation des territorialités locales » Scientia Canadensis, vol. 35, n 1-2, p. 65-83. 
CRONON, W., 2009 « Le problème de la wilderness ou le retour vers une mauvaise nature », Écologie et politique, Vol. 38, n 1, p. 173-199.

Debarbieux, B., 2014 [5e éd.], " Les problématiques de l'image et de la représentation en géographie », dans Bailly, A. (dir.), Les concepts de la géographie humaine, Paris, Armand Colin, p. 199-212.

DE KOnINCK, R., 2009, Le monde à la carte (5e éd., Montréal, Presses Interuniversitaires, 213 pages.

DesBIENS, C., 2015, Puissance Nord : Territoire, identité et culture de l'hydroélectricité au Québec, Québec, Presses de l’Université Laval, 318 pages.

Desbiens, C., 2012, « 10 Idées pour le Nord : un manifeste pour la nordicité », Cahiers de géographie du Québec, vol. 56, n 159, p. 643-659

Desroches, Ph. et Freedman, M., 2012, « La participation des communautés locales dans les parcs nationaux au nord du 49e parallèle », Téoros. Revue de recherche en tourisme, vol. 31, $\mathrm{n}^{\circ}$ 1, p. 124-126.

DoRAIS, L.-J., 2008, « Terre de l'ombre ou terre d'abondance ? Le Nord des Inuit », dans Chartier, D. (dir.) Le(s) Nord(s) imaginaire(s), Montréal, Presses de l’Université du Québec, p. 9-22.

Evernden, N., 1992, The Social Creation of Nature, Baltimore, Johns Hopkins Press, 179 pages.

FARGET, D. ET FULLUM-LAVERY, M., 2014, « La place réservée à l'avis des peuples autochtones dans le cadre du processus de prise de décision concernant le Plan Nord ou l'exploitation du Nord québécois », Revue de droit de McGill /McGill Law Journal, vol. 59, n³, p. 595-653.

GERMAIN, A., 2012, "Le fédéralisme et la territorialité : l'état québécois, les autochtones et le pluralisme territorial », Les Cahiers de la CREQC (Chaire de recherche du Canada en études québécoises et canadiennes, $\mathrm{n}^{\circ} 1,22$ pages.

GIRARD, C. ET BRISSON, C., 2018, Reconnaissance et exclusion des peuples autochtones au Québec. Du traité d'alliance de 1603 à nos jours, Québec, Presses de l'Université Laval, 265 pages.

GLON, É., 2012, « Cartographie participative autochtone et réappropriation culturelle et territoriale ", Espace, populations, sociétés, $\mathrm{n}^{\circ} 1$ [En ligne] URL : http://journals.openedition.org/eps/4827, consulté le 05 septembre 2018.

Gouvernement Du QuebeC, 2015, Le Plan Nord à l'horizon 2035 : plan d'action 2015-20, Québec, Secrétariat au Plan Nord, 112 pages.

Gouvernement Du QueBEC, 2011, Plan Nord : faire le nord ensemble, le chantier d'une génération, Québec, Ministère des ressources naturelles et de la faune, 172 pages.

HAmeliN, L.-E., 2000, « Le Nord et l'hiver dans l'hémisphère boréal », Cahiers de géographie du Québec, vol. 44, n 121, p. 5-25.

Hamelin, L.-E., 1975, Nordicité canadienne, Montréal, Hurtubise, 302 pages.

Hamelin, L.-E., 1973, Le Mushuau Nipi à l'âge du caribou (Nouveau-Québec. Québec, Centre d'Études nordiques, Université Laval, 109 pages.

Hertzog, A., Paris, F. Et Perret, O., 2011, " La planète habitée », dans Sierra, Ph., dir. La Géographie : concepts, savoirs et enseignements, Paris, Armand Colin, p. 85-104

IANKOVA, K., 2005, «Le tourisme autochtone au Québec », Globe - revue internationale d'études québécoises, vol. 8, n 1, p. 85-98.

ISQ, 2011, Population totale, superficie et densité du Nord-du-Québec, Institut de la statistique du Québec, [en ligne] URL http://www.stat.gouv.qc.ca/statistiques/recensement/2011/recens2011_10/population/popt ot_superficie10.htm, consulté le 12 septembre 2018.

Joliet, F. Eт JACOBS, P., 2009, « Le Wilderness, une manière de voir et d'être à la nature sauvage : le prisme paysager de Tremblant Québec », Cahiers de géographie du Québec, vol. 53, n 
148 , p. $27-46$

Knotsch, C., Siebenmorgen, P. et BradshaW, B., 2010, "Les Ententes sur les répercussions et les avantages et le bien-être des communautés : Des occasions ratées ? ", Recherches amérindiennes au Québec, vol. 40, n³, p. 59-68.

LACASSE, J.-P., 1996, « Le territoire dans l'univers innu d'aujourd'hui », Cahiers de géographie du Québec, vol. 40, n 110, p. 185- 204

LEVY, J., 2008, « La géographie culturelle a-t-elle un sens ? », Annales de géographie, n 660-661, p. $27-46$.

MANN, C. C., 2007, 1491. Nouvelles révélations sur les Amériques avant Christophe Colomb, Paris, Albin Michel, 480 pages.

Mercier, G. et Bethemont, J., dir., 1998, La ville en quête de nature, Québec, Septentrion, Coll. Les Nouveaux Cahiers du CÉLAT, n²1, 253 pages.

MorisSONNEAU, C., 1978, La terre promise : le mythe du Nord québécois, Montréal, Hurtubise, 212 pages.

MoumANEIX, C., 2012, «Fédéralisme, nature et gestion des communautés dans les parcs nationaux en Amérique du nord (Canada, États-Unis) », Études canadiennes, n 72, 2012, p. 141-159.

PAKSY, M., 2016, "Droit et géographie », Géographie et cultures, n 100 [En ligne] URL : http://journals.openedition.org/gc/4701, consulté le 29 août 2018.

PollinI, A., 2012, « La représentation de l'espace chez Hérodote », dans Guisard, P. et Laizé, C. (dir.), Expériences et représentations de l'espace, Paris, Ellipses, p. 109-124.

POLLOCK, R., 2008, «ReVisiting the North: Reflections from the Mushuau-nipi (George River) », International Journal of Canadian Studies, $\mathrm{n}^{\circ}$. 38, p. 61-90.

Posthumus, S., 2012, "Penser l'imagination environnementale française sous le signe de la différence ", Raison publique, ${ }^{\circ} 17, \mathrm{p} .15-31$.

RIVARD, É. et DesBiens, C., 2011, « Le Plan Nord, monstre à deux têtes et autres chimères géographiques », Recherches amérindiennes au Québec, vol. 41, n 1, p. 83-89.

Roche-Eley, D., 1979, Notre Pays: The Signification of the Wilderness Image in the Quebec Cinema During the Quiet Revolution, Thèse de doctorat, Département des communications, Université McGill, Montréal, 534 pages.

SCHNEIDER, N., 2013, " Pèlerinage au Nitassinan », Géopleinair, [en ligne] URL : http://www.geopleinair.com/destinations/regions/hors-quebec/pelerinage-au-nitassinan/, consulté le 7 septembre 2018

SIMARD, M., 2012, " Urbain, rural et milieux transitionnels. Les catégories géographiques de la ville diffuse », Cahiers de géographie du Québec, vol. 56, n 157, p. 109-124.

SIMARD, M., MALTAIS, E. et BRISSON, C., 2018, « Les représentations sociospatiales des travailleurs faisant la navette par avion vers le Nord du Québec », Espace, populations, sociétés, $\mathrm{n}^{\circ} 3$ (à paraître).

SiouI, M.-M., 2018, « Ungava, loin de l'urne et loin des intérêts », Le Devoir, édition du 8 septembre 2018, URL : https:/www.ledevoir.com/politique/quebec/536262/ungava-loin-de-l-urne-etloin-des-interets, consulté le 9 septembre 2018.

Statistique CANADA, 2018, Perspective et mesure de l'urbain, [En ligne] URL : https://www150.statcan.gc.ca/n1/pub/92f0138m/92f0138m2009001-fra.htm, consulté le 6 septembre 2018.

TESSIER, J.-L., 2004, « Oekoumène », Hypergéo - Encyclopédie électronique de géographie [en ligne] URL : http://www.hypergeo.eu/spip.php?article382, consulté le 27 août 2018. 